Bull. Austral. Math. Soc.

20F15, 20A18, 20A20, 03C65

Vol. 53 (1996) [425-439]

\title{
COMMENSURABILITY AND ELEMENTARY EQUTVALENCE OF POLYCYCLIC GROUPS
}

\author{
Deborah Raphael
}

\begin{abstract}
It is shown that two polycyclic-by-finite groups $G$ and $H$, satisfying the same sentences with one alternation of quantifiers, are commensurable. In fact we show something stronger: given $n>1$ there is a subgroup $H_{n}$ of $H$ and a subgroup $G_{n}$ of $G$ such that $H_{n} \simeq G, G_{n} \simeq H$ and the indices $\left[G: G_{n}\right]$ and $\left[H: H_{n}\right.$ ] are finite and prime to $n$.
\end{abstract}

\section{INTRODUCTION}

This work is motivated by a problem proposed by G. Sabbagh: to find an algebraic characterisation of elementary equivalence in the class of polycyclic-by-finite groups. F.Oger solved this problem for finitely generated finite-by-nilpotent groups; in [7], he proves that two finitely generated finite-by-nilpotent groups $G$ and $H$ satisfy the same sentences if and only if $G \times \mathbb{Z} \simeq H \times \mathbb{Z}$. This result can not be generalised to polycyclicby-finite groups: there exist polycyclic-by-finite groups $G$ and $H$ such that $G \equiv H$ and $G \times \mathbb{Z} \not \boldsymbol{H} \times \mathbb{Z}$. Here we give a necessary condition for any two polycyclic-by-finite groups to satisfy the same sentences with one alternation of quantifiers.

THEOREM. Let $G$ and $B$ be polycyclic-by-finite groups satisfying the same sentences with one alternation of quantifiers. Given an integer $r>1$, there is a subgroup $H_{r}$ of $H$ and a subgroup $G_{r}$ of $G$ such that $H_{r} \simeq G, G_{r} \simeq H$ and the indices $\left[G: G_{r}\right.$ ] and $\left[H: H_{r}\right]$ are finite and prime to $r$.

This Theorem is a generalisation of [5], where Oger proves the same result in the case $G$ and $H$ are finitely generated finite-by-nilpotent groups. The techniques used in [5] do not work for polycyclic-by-finite groups and we do not suppose this result known in the proof of the Theorem.

The aim of Sections 2, 3 and 4 is the proof of the Theorem. In Section 2, we prove a few lemmas concerning subgroups defined by formulas. Section 3 deals only with polycyclic-by-finite groups: we give a sufficient condition for a subgroup to have finite index prime to a given integer and we show how to arrive at this condition applying

Received 27th July, 1995

I am in debt to $F$. Oger for many helpful conversations and suggestions.

Copyright Clearance Centre, Inc. Serial-fee code: 0004-9729/96 \$A2.00+0.00. 
the lemmas of Section 2. All we need in the proof of the Theorem is given in this section; the proof itself is given in Section 4. In Section 5 we discuss the connections between some equivalence relations in the class of polycyclic-by-finite groups (the two equivalence relations appearing in the Theorem, commensurability, 三, "having the same finite images"); in particular, we give an example showing that the converse of the Theorem is not true.

[12] is our basic reference for polycyclic-by-finite groups. If $G$ is a group, $B<G$ means that $H$ is a subgroup of $G$; if $n \geqslant 1$ is an integer, $G^{n}$ is the subgroup $\left\langle g^{n}\right|$ $g \in G\rangle ; G^{\prime}$ is the derived subgroup $[G, G] ; \zeta(G)$ denotes the centre of $G ; \mathcal{F}(G)$ is the set of isomorphism classes of the finite images of $G$; if $G$ is polycyclic-by-finite, $h(G)$ is the Hirsch number of $G$.

The definitions of formula (existential, universal, quantifier free), sentence and language can be found in [2]. The formulas considered here are in the first-order language of groups, $L=\left\{\cdot, *^{-1}, 1\right\}$, where is a binary function for the group operation, $*^{-1}$ is an unary fuction for the inverse and 1 is a constant for the identity element.

\section{Defin ABILITY}

A formula $\phi$ with one alternation of quantifiers can be written either in the form $\left(\exists X_{1}\right) \ldots\left(\exists X_{n}\right)\left(\forall Y_{1}\right) \ldots\left(\forall Y_{m}\right) \theta$, or in the form $\left(\forall Y_{1}\right) \ldots\left(\forall Y_{m}\right)\left(\exists X_{1}\right) \ldots\left(\exists X_{n}\right) \theta$, where $\theta$ is a formula without quantifiers. The first one is called an $\exists \forall$ formula and the last one a $\forall \exists$ formula. Given a formula $\phi\left(X_{1}, \ldots, X_{n}\right)$ with $n$ free variables and a group $G$ with $g_{1}, \ldots, g_{n}$ in $G$, we say that $g_{1}, \ldots, g_{n}$ satisfy $\phi$ in $G$, and we write $G \vDash \phi\left(g_{1}, \ldots, g_{n}\right)$, if $\phi\left(g_{1}, \ldots, g_{n}\right)$ is true in $G$. Given groups $G$ and $H$ we write $G \equiv H$ if $G$ and $H$ are elementary equivalent; we write $G \equiv_{1} H$ if $G$ and $H$ satisfy the same sentences with one alternation of quantifiers.

DEFINITION: Let $\phi$ be a formula with one free variable and let $G$ be a group. We denote by $G_{\phi}$ the set $\{g \in G \mid G \vDash \phi(g)\}$. Clearly, $G_{\phi}$ is a subgroup of $G$ if and only if $\phi(1)$ and $(\forall X)(\forall Y)\left((\phi(X) \wedge \phi(Y)) \rightarrow \phi\left(X Y^{-1}\right)\right)$ are true in $G$. In this case, we say that $\phi$ defines the subgroup $G_{\phi}$ in $G$.

From now on we shall often make use of formulas with one free variable defining subgroups in a given group $G$. When it is clear from the context that the formula must have one free variable we do not state this hypothesis explicitly.

LEMMA 2.1. Let $\phi$ and $\psi$ be formulas. The following four statements hold.

(i) For any group $G$, if $G_{\phi}$ is a subgroup of $G$ then $G_{\phi}$ is a characteristic subgroup of $G$ (in particular, $G_{\phi} \triangleleft G$ ).

(ii) For any group $G$ such that $G_{\phi}$ and $G_{\psi}$ are subgroups of $G$, we have $G_{(\phi \wedge \psi)}=G_{\phi} \cap G_{\psi}$. 
(iii) There is a formula $\gamma$ such that $G_{\gamma}=G_{\phi} G_{\psi}$, for any group $G$ in which $G_{\phi}$ and $G_{\psi}$ are subgroups. If $\phi$ and $\psi$ are existential, so is $\gamma$.

(iv) If $\phi$ and $\psi$ are existential, then there is an existential formula $\lambda$ such that $G_{\lambda}=\left(G_{\phi}\right)_{\psi}$, for any group $G$ with $G_{\phi}$ subgroup of $G$ and $\left(G_{\phi}\right)_{\psi}$ subgroup of $G_{\phi}$.

Proof: In order to prove (i), it suffices to observe that formulas are preserved by automorphisms (and in particular by the inner automorphisms). The proof of (ii) is straightforward. For (iii), set $\gamma(Z)$ equal to $(\exists X)(\exists Y)(\phi(X) \wedge \psi(Y) \wedge(Z=X Y))$.

To prove (iv), we write $\psi$ in the form $\left(\exists Y_{1}\right) \ldots\left(\exists Y_{n}\right) \psi^{\prime}\left(Y_{1}, \ldots, Y_{n}, Y\right)$, where $\psi^{\prime}$ is a formula without quantifiers. The statement follows easily if we set $\lambda(Z)$ equal to

$$
\left(\exists Z_{1}\right) \ldots\left(\exists Z_{n}\right)\left(\phi(Z) \wedge \bigwedge_{i=1}^{n} \phi\left(Z_{i}\right) \wedge \psi^{\prime}\left(Z_{1}, \ldots, Z_{n}, Z\right)\right) .
$$

The fact that subgroups defined by formulas are normal (Lemma 2.1 (i)) will often be used without any further comment.

LEMma 2.2. Let $\phi$ and $\psi$ be existential formulas and let $G$ and $H$ be groups such that $G \equiv_{1} H$. The following statements hold:

(i) $G_{\phi} \triangleleft G$ if and only if $H_{\phi} \triangleleft H$.

(ii) $G_{\psi} \subset G_{\phi}$ if and only if $H_{\psi} \subset H_{\phi}$.

Proof: Since $\phi$ is existential, $\phi(1)$ and $(\forall X)(\forall Y)\left((\phi(X) \wedge \phi(Y)) \rightarrow \phi\left(X Y^{-1}\right)\right)$ are $\forall \exists$ formulas. This proves (i). For any group $K$, the sentence $(\forall X)(\psi(X) \rightarrow \phi(X))$ is satisfied in $K$ if an only if $K_{\psi} \subset K_{\phi}$. Statement (ii) follows from the fact that this sentence is $\forall \exists$ if $\psi$ and $\phi$ are existential.

In Chapter 3, we deal with several situations in which we have to describe finite groups by formulas. In order not to repeat slighty variations of the same argument, we state Lemma 2.3 bellow.

LEMMA 2.3. Let $\phi$ and $\psi$ be formulas and let $G$ be a group such that $G_{\psi}$ and $G_{\phi}$ are both subgroups of $G$ with $G_{\psi} \subset G_{\phi}$ and $\left|G_{\phi} / G_{\psi}\right|=n$ finite. Then, there is a formula $\beta\left(X_{1}, \ldots, X_{n}\right)$ satisfying the following two conditions.

(i) For every group $H$ with subgroups $H_{\phi}$ and $H_{\psi}$ such that $H_{\psi} \subset H_{\phi}$, we have $H \vDash \beta\left(b_{1}, \ldots, b_{n}\right)$ if and only if $\left\{b_{1} H_{\psi}, \ldots, b_{n} H_{\psi}\right\}$ is a subgroup of $H_{\phi} / H_{\psi}$ isomorphic to $G_{\phi} / G_{\psi}$.

(ii) If $\phi$ and $\psi$ are existential then $\beta$ is $\exists \forall$; if $\phi$ and $\psi$ are quantifier free formulas then $\beta$ is quantifier free.

Moreover, if $\phi$ and $\psi$ are existential and $H$ is a group with $G \equiv_{1} H$ we have also:

(iii) $G_{\phi} / G_{\psi} \simeq H_{\phi} / H_{\psi}$;

(iv) $\quad H \vDash \beta\left(b_{1}, \ldots, b_{n}\right)$ if and only if $\left\{b_{1} H_{\psi}, \ldots, b_{n} H_{\psi}\right\}=H_{\phi} / H_{\psi}$. 
Proof: Set $G_{\phi} / G_{\psi}=\left\{a_{1} G_{\psi}, \ldots, a_{n} G_{\psi}\right\}$ and let $\sigma:\{1, \ldots, n\} \times\{1, \ldots, n\} \longrightarrow$ $\{1, \ldots, n\}$ be such that $a_{i} a_{j} a_{\sigma(i, j)}^{-1} \in G_{\psi}$ for $1 \leqslant i, j \leqslant n$. Denote by $S_{n}$ the permutation group of $\{1, \ldots, n\}$ and define $\beta\left(X_{1}, \ldots, X_{n}\right)$ as:

$$
\overbrace{\left(\bigwedge_{1 \leqslant i \leqslant n} \phi\left(X_{i}\right)\right) \wedge\left(\bigwedge_{1 \leqslant i<j \leqslant n} \neg \psi\left(X_{i} X_{j}^{-1}\right)\right)}^{\beta_{1}\left(X_{1}, \ldots, x_{n}\right)} \wedge \overbrace{\left(\bigvee_{\pi \in S_{n}} \bigwedge_{1 \leqslant i, j \leqslant n} \psi\left(X_{\pi(i)} X_{\pi(j)} X_{\pi \circ \sigma(i, j)}^{-1}\right)\right)}^{\beta_{2}\left(x_{1}, \ldots, x_{n}\right)}
$$

Proof of (i). Suppose $H$ is a group in which $H_{\phi} \subset H_{\psi}$ are both subgroups of $H$ and let $b_{1}, \ldots, b_{n}$ be in $H$. By interpretating $\beta_{1}\left(b_{1}, \ldots, b_{n}\right)$ and $\beta_{2}\left(b_{1}, \ldots, b_{n}\right)$ in $H$, we conclude that $H \vDash \beta\left(b_{1}, \ldots, b_{n}\right)$ if and only if the two following conditions hold:

(a) $\left\{b_{1} H_{\psi}, \ldots, b_{n} H_{\psi}\right\}$ is a subset of $H_{\phi} / H_{\psi}$ which has $n$ elements.

(b) There is $\pi \in S_{n}$ such that $b_{i} b_{j} H_{\psi}$ is equal to $b_{\pi \circ \sigma(i, j)} H_{\psi}$, for $1 \leqslant i, j \leqslant n$. So, if $H \vDash \beta\left(b_{1}, \ldots, b_{n}\right)$, by (a) we can define the function $f_{\pi}: G_{\phi} / G_{\psi} \rightarrow H_{\phi} / H_{\psi}$ given by the equalities $f_{\pi}\left(a_{i} G_{\psi}\right)=b_{\pi(i)} H_{\psi}$, for $1 \leqslant i \leqslant n$; moreover, this function is injective and $\Im(f)$ is $\left\{b_{1} H_{\psi}, \ldots, b_{n} H_{\psi}\right\}$. By (b) we know that $f_{\pi} \in \operatorname{Hom}\left(G_{\phi} / G_{\psi}, H_{\phi} / H_{\psi}\right)$. Therefore, $\left\{b_{1} H_{\psi}, \ldots, b_{n} H_{\psi}\right\}$ is a subgroup of $H_{\phi} / H_{\psi}$ isomorphic to $G_{\phi} / G_{\psi}$.

Conversely, suppose $\left\{b_{1} H_{\psi}, \ldots, b_{n} H_{\phi}\right\}$ is a subgroup of $H_{\phi} / H_{\psi}$ isomorphic to $G_{\phi} / G_{\psi}$. It is clear that (a) holds. So, we only have to prove that (b) holds. Let $f: G_{\phi} / G_{\psi} \hookrightarrow H_{\phi} / H_{\psi}$ be a monomorphism whose image is $\left\{b_{1} H_{\psi}, \ldots, b_{n} H_{\psi}\right\}$ and take $\pi \in S_{n}$ such that $f\left(a_{i} G_{\psi}\right)=b_{\pi(i)} H_{\psi}$ for $1 \leqslant i \leqslant n$. Since $a_{i} a_{j} G_{\psi}$ is equal to $a_{\sigma(i, j)} G_{\psi}$, $f$ sends $a_{i} a_{j} G_{\psi}$ to $b_{\pi \circ \sigma(i, j)} H_{\psi}$. As $f$ is a group homomorphism we conclude that $b_{i} b_{j} H_{\psi}$ is equal to $b_{\pi \circ \sigma(i, j)} H_{\psi}$ for $1 \leqslant i, j \leqslant n$. Then (b) is true and (i) is proved.

Proof of (ii). This is a direct consequence of the definition of $\beta$.

Proof of (iii). Suppose that $G \equiv_{1} H$. Recall that, by Lemma 2.2, $H_{\psi} \subset H_{\phi}$ and they are both normal subgroups of $H$. From (i) and (ii) we know that $G$ satisfies the $\exists \forall$ sentence $\left(\exists X_{1}\right) \ldots\left(\exists X_{n}\right) \beta$. Then, there are $b_{1}, \ldots, b_{n}$ such that $B \vDash \beta\left(b_{1}, \ldots, b_{n}\right)$. Again by (i), there is a group monomorphism from $G_{\phi} / G_{\psi}$ to $H_{\phi} / H_{\psi}$. So, to prove that these two finite quotient groups are isomorphic, it is enough to show that $\left|H_{\phi} / H_{\psi}\right| \leqslant n$. Consider the sentence $\theta$ given by:

$$
\left(\forall X_{1}\right) \ldots\left(\forall X_{n+1}\right)\left(\bigwedge_{i=1}^{n+1} \phi\left(X_{i}\right) \longrightarrow\left(\bigvee_{1 \leqslant i<j \leqslant n+1} \psi\left(X_{i} X_{j}^{-1}\right)\right)\right)
$$

When interpreted in a group $K$ such that $K_{\psi} \subset K_{\phi}$ are both subgroups of $K, \theta$ says " $K_{\phi} / K_{\psi}$ has $\leqslant n$ elements". The sentence $\theta$ is $\forall \exists$ and $G \vDash \theta$. Therefore, if $H \equiv_{1} G$ then $H$ satisfies $\theta$, and so, $\left|H_{\phi} / H_{\phi}\right| \leqslant n$.

Proof of (iv). Since $\left|G_{\phi} / G_{\psi}\right|=n$, (iv) is a direct consequence of (i) and (iii). 
Corollary 2.4. Let $L$ be a finite group and suppose $|L|=n$. Then, there exists a quantifier free formula $\beta_{L}\left(X_{1}, \ldots, X_{n}\right)$ and an universal formula $\rho_{L}\left(X_{1}, \ldots, X_{n}\right)$ such that, for every group $B$, the following statements hold:

(1) $B \vDash \beta_{L}\left(h_{1}, \ldots, h_{n}\right)$ if and only if $\left\{h_{1}, \ldots, b_{n}\right\}$ is a subgroup of $H$ isomorphic to $L$.

(2) $H \vDash \rho_{L}\left(h_{1}, \ldots, h_{n}\right)$ if and only if $\left\{h_{1}, \ldots, b_{n}\right\}$ is a normal subgroup of $H$ isomorphic to $L$.

Proof: (i) In Lemma 2.3, take $X=X$ and $X=1$ as being $\phi(X)$ and $\psi(X)$ respectively, and take $G$ equals to $L$. Now, for every group $H, H_{\phi}=B$ and $H_{\psi}=1$. By Lemma 2.3-(iii), the formula $\beta\left(X_{1}, \ldots, X_{n}\right)$ is quantifier-free. Taking $\beta_{L}\left(X_{1}, \ldots, X_{n}\right)$ equal to the this formula, Lemma 2.3-(ii) proves (i).

(ii) Take $\rho_{L}(X)$ as $(\forall X)\left(\left(\bigwedge_{1 \leqslant i \leqslant n 1 \leqslant j \leqslant n} X X_{i} X^{-1}=X_{j}\right) \wedge \beta_{L}(\bar{X})\right)$, where $\bar{X}$ is $\left(X_{1}, \ldots, X_{n}\right)$. We have added to the formula $\beta_{L}$ a formula saying "the conjugate of $X_{i}$ by any element is among $X_{1}, \ldots, X_{n}$ ". Now (ii) follows from (i).

Corollary 2.5. Let $\phi$ and $\psi$ be existential formulas and let $G$ be a group such that $G_{\psi}$ and $G_{\phi}$ are both subgroups of $G$. Suppose that $G_{\psi} \subset G_{\phi}$ and that $\left|G_{\phi} / G_{\psi}\right|$ is finite. Let $y_{1}, \ldots, y_{k}$ be elements of $G$ such that $G_{\phi} \subset\left\langle y_{1}, \ldots, y_{k}\right\rangle G_{\phi}$. Then, there exists an $\exists \forall$ formula $\gamma\left(Y_{1}, \ldots, Y_{k}\right)$ satisfying the following two conditions:

(i) $G \vDash \gamma\left(y_{1}, \ldots, y_{k}\right)$;

(ii) $H \vDash \gamma\left(z_{1}, \ldots, y_{k}\right)$ implies $H_{\phi} \subset\left\langle z_{1}, \ldots, z_{k}\right\rangle H_{\psi}$, for every group $H$ with $G \equiv_{1} H$.

Proof: Put $n=\left|G_{\phi} / G_{\psi}\right|$ and consider the $\exists \forall$ formula $\beta\left(X_{1}, \ldots, X_{n}\right)$ given in Lemma 2.3. Item (iv) of the same lemma implies that, for every group $H$ with $G \equiv_{1} H$, we have: $\beta\left(b_{1}, \ldots, b_{n}\right)$ is true in $H$ if and only $\left\{b_{1} H_{\psi}, \ldots, b_{n} H_{\psi}\right\}$ is equal to $H_{\phi} / H_{\psi}$.

Let $a_{1}, \ldots, a_{n}$ in $G$ be such that $G_{\phi} / G_{\psi}=\left\{a_{1} G_{\psi}, \ldots, a_{n} G_{\psi}\right\}$, and set $\bar{y}=$ $\left(y_{1}, \ldots, y_{k}\right)$ and $\bar{Y}=\left(Y_{1}, \ldots, Y_{k}\right)$. Since $G_{\phi} \subset\left(y_{1}, \ldots, y_{k}\right) G_{\psi}$, there are $n$ words, $u_{1}(Y), \ldots, u_{n}(\bar{Y})$, such that $u_{1}(\bar{y}) G_{\psi}=a_{1} G_{\psi}, \ldots, u_{n}(\bar{y}) G_{\psi}=a_{n} G_{\psi}$. Define $\gamma(Y)$ as being $\beta\left(u_{1}(\bar{Y}), \ldots, u_{n}(\bar{Y})\right)$. This formula is $\exists \forall$ because $\beta$ is $\exists \forall$. As $\beta\left(a_{1}, \ldots, z_{n}\right)$ is true in $G$, we know that $G \vDash \gamma(\bar{y})$. This proves (i).

Now, let $H$ be a group for which $G \equiv_{1} H$ and let $\bar{z}=\left(z_{1}, \ldots, z_{k}\right)$ be such that $H \vDash \gamma(\bar{z})$. Then, $H \vDash \beta\left(u_{1}(\bar{z}), \ldots, u_{n}(\bar{z})\right)$ and we have that $H_{\phi} / H_{\psi}$ is equal to $\left\{u_{1}(\bar{z}) H_{\psi}, \ldots, u_{n}(\bar{z}) H_{\psi}\right\}$. Consequently, $H_{\phi}$ is contained in $\left\langle z_{1}, \ldots, z_{k}\right\rangle H_{\psi}$.

\section{POLYCYCLIC-BY-FINITE GROUPS}

We begin this section with a result on polycyclic-by-finite groups that gives a sufficient condition for a subgroup to have finite index prime to a given integer. 
Proposition 3.1. Consider a polycyclic-by-finite group $G$, an integer $c \geqslant 1$ and a chain of normal subgroups of $G, 1=G_{c+1} \triangleleft G_{c} \triangleleft \cdots \triangleleft G_{1} \triangleleft G$, such that $G / G_{1}$ is finite and $G_{i} / G_{i+1}$ is $A$ belian for $1 \leqslant i \leqslant c$. Suppose $K$ is a subgroup of $G$ and $r \geqslant 1$ is an integer. If $G=K G_{1}$ and $G_{i} \subset K G_{i}^{r}$, for $1 \leqslant i \leqslant c$, then $[G: K]$ is finite and prime to $r$.

Proof: We first treat the Abelian case.

(i) Given finitely generated Abelian groups $B<A$ and an integer $r>1$, if $A=$ $B A^{r}$, then $[A: B]$ is finite and prime to $r$.

Given a finitely generated Abelian group $H$, write $H$ as the direct sum of a free Abelian group and a finite group. Now it is easy to show that, if $H^{r}=H$, then $|H|$ is finite and prime to $r$. Setting $H$ equal to $A / B$, (i) follows.

We prove the proposition by induction on $c$. If $c=1$, then $G_{1}$ is Abelian and $G_{1}=K G_{1}^{r} \cap G_{1}=\left(K \cap G_{1}\right) G_{1}^{r}$; from (i), it follows that $\left[G_{1}: K \cap G_{1}\right]$ is finite and prime to $r$. Since $G=K G_{1}$ and $G_{1}$ is normal in $G$, then $[G: K]$ is $\left[G_{1}: K \cap G_{1}\right]$. Now suppose $c>1$. Set $\bar{K}=K G_{c} / G_{c}, \bar{G}=G / G_{c}$ and $\overline{G_{i}}=G_{i} / G_{c}$, for $1 \leqslant i \leqslant c$. By the induction hypothesis, we know that $[\bar{G}: \bar{K}]$ is finite and prime to $r$. As $[\bar{G}: \bar{K}]$ is equal to $\left[G: K G_{c}\right]$, it is enough to prove that $\left[K G_{c}: K\right]$ is finite and prime to $r$. The subgroup $G_{c}$ is finitely generated Abelian and $G_{c}=K G_{c}^{r} \cap G_{c}=\left(K \cap G_{c}\right) G_{c}^{r}$. Therefore, by (i), the index $\left[G_{c}: K \cap G_{c}\right]$ is finite and prime to $r$. As $\left[K G_{c}: K\right]$ is equal to $\left[G_{c}: K \cap G_{c}\right]$, the proposition is proved.

In the proof of the Theorem, one of the things we need is: a $\exists \forall$ formula $\gamma$ with $n$ free variables and such that if $H \vDash \gamma\left(h_{1}, \ldots, h_{k}\right)$ then $H_{r}=\left\langle h_{1}, \ldots, h_{k}\right\rangle$ is a subgroup of $\boldsymbol{H}$ whose index is finite and prime to $r$. We shall use the proposition above to achieve this. So, the next results clear the way to build a formula implying the proposition's hypothesis. The formula itself is given in Corollary 3.6 , at the end of this section. We recall two results that allow us to describe certain subgroups by existential formulas:

(i) Let $G$ be a polycyclic group. There exists a positive integer $k$ such that every $g$ belonging to $G^{\prime}$ can be written as a product $\left[g_{1}, g_{2}\right] \cdots\left[g_{2 k-1}, g_{2 k}\right]$, where $g_{1}, \ldots, g_{2 k}$ are in $G$.

(ii) Let $G$ be a polycyclic-by-finite group and let $m \geqslant 1$ be an integer. There is an integer $r$ such that every $g$ in $G^{m}$ can be written as $g=g_{1}^{m} \cdots g_{r}^{m}$, with $g_{1}, \ldots, g_{r}$ in $G$.

The first result is a consequence of a theorem proved by Rhemtulla in [10]. A proof of (ii) can be found in [6]. See also [11], where Romankov generalises these results to any verbal subgroup provided the group $G$ is polycyclic. A direct consequence of these results is the following lemma. 
LEMmA 3.2. (i) If $K$ and $H$ are polycyclic groups, there is an existential formula $\lambda$ such that $K_{\lambda}=K^{\prime}$ and $H_{\lambda}=H^{\prime}$.

(ii) If $K$ and $H$ are polycyclic-by-finite groups and $m>1$ is an integer, there is an existential formula $\gamma$ such that $K_{\gamma}=K^{m}$ and $H_{\gamma}=H^{m}$.

Proof: (i) Define $\lambda(X)$ as $\left(\exists Y_{1} \ldots \exists Y_{2 l}\right)\left(X=\left[Y_{1}, Y_{2}\right] \cdots\left[Y_{2 l-1}, Y_{2 l}\right]\right)$, where each element of $H^{\prime}$ and each element of $K^{\prime}$ is a product of $l$ commutators.

(ii) Similarly, define $\gamma(X)$ as $\left(\exists Y_{1} \ldots \exists Y_{s}\right)\left(X=Y_{1}^{m} \cdots Y_{s}^{m}\right)$, where each element of $H^{m}$ and each element of $K^{m}$ is a product of $s m$-th powers.

Now we apply the results of the preceding section to polycyclic-by-finite groups. In order to make notation lighter we have chosen to write $G_{\phi}^{m}$ instead of $\left(G_{\phi}\right)^{m}$ (where $G$ is a group, $m$ is an integer and $\phi$ is a formula).

Lemma 3.3. Let $G$ and $B$ be polycyclic-by-finite groups such that $G \equiv_{1} H$. Let $\psi$ and $\phi$ be existential formulas such that $G_{\psi}$ and $G_{\phi}$ are subgroups of $G$ with $G_{\psi} \subset G_{\phi}$. If $G_{\phi} / G_{\psi}$ is $A$ belian then $H_{\phi} / H_{\psi}$ is isomorphic to $G_{\phi} / G_{\psi}$.

Proof: Before beginning the proof, we remark that $H_{\phi} / H_{\psi}$ makes sense since, by Lemma 2.2, $H_{\psi} \subset H_{\phi}$ and these two sets are normal subgroups of $H$. The sentence $(\forall X)(\forall Y)\left((\phi(X) \wedge \phi(Y)) \rightarrow \psi\left(X Y X^{-1} Y^{-1}\right)\right)$ is $\forall \exists$; this sentence means " $K_{\phi} / K_{\psi}$ is Abelian" in any group $K$ for which $K_{\psi} \triangleleft K, K_{\psi} \triangleleft K$ and $K_{\psi} \subset K_{\phi}$. Thus, $G_{\phi} / G_{\psi}$ Abelian implies $H_{\phi} / H_{\psi}$ Abelian.

Two finitely generated Abelian groups $A$ and $B$ are isomorphic if and only if, for each integer $m>1$, the finite groups $A / A^{m}$ and $B / B^{m}$ are isomorphic. Moreover, we have:

$$
\left(G_{\phi} / G_{\psi}\right) /\left(G_{\phi} / G_{\psi}\right)^{m} \simeq G_{\phi} /\left(G_{\psi} G_{\phi}^{m}\right) \text { and }\left(H_{\phi} / H_{\psi}\right) /\left(H_{\phi} / H_{\psi}\right)^{m} \simeq H_{\phi} /\left(H_{\psi} H_{\phi}^{m}\right)
$$

Thus, it is enough to show that $G_{\phi} /\left(G_{\psi} G_{\phi}^{m}\right) \simeq H_{\phi} /\left(H_{\psi} H_{\phi}^{m}\right)$ for each $m>1$.

It follows from Lemma 3.2 and Lemma 2.1-(iii) and (iv) that, for each $m>1$, there is an existential formula $\theta$ such that $G_{\theta}=G_{\psi} G_{\phi}^{m}$ and $H_{\theta}=H_{\psi} H_{\phi}^{m}$. As $G_{\phi} / G_{\theta}$ and $H_{\phi} / H_{\theta}$ are finite, Lemma 2.3 yields $G_{\phi} / G_{\theta} \simeq H_{\phi} / H_{\theta}$.

Proposition 3.4. Let $G$ and $H$ be polycyclic-by-finite groups such that $G \equiv_{1} H$. Then, there are two positive integers $m$ and $c$ and there are existential formulas $\phi^{1}, \ldots, \phi^{c+1}$ such that $1=G_{\phi^{c+1}} \triangleleft G_{\phi^{c}} \triangleleft \cdots \triangleleft G_{\phi^{1}}=G^{m}$ and $1=$ $H_{\phi^{c+1}} \triangleleft H_{\phi^{c}} \triangleleft \cdots \triangleleft H_{\phi^{1}}=H^{m}$ are the derived series of $G^{m}$ and $H^{m}$. Moreover, $G_{\phi^{i}} / G_{\phi^{i+1}} \simeq H_{\phi^{i}} / H_{\phi^{i+1}}$, for $1 \leqslant i \leqslant c$. 
Proof: There is an integer $m>1$ such that $G^{m}$ and $H^{m}$ are polycyclic and therefore soluble. Let $c \geqslant 1$ be the minimal integer such that $G^{m}$ and $H^{m}$ are soluble of class $\leqslant c$. Consider $1=G_{c+1} \triangleleft G_{c} \triangleleft \cdots \triangleleft G_{1}=G^{m}$ and $1=H_{c+1} \triangleleft H_{c} \triangleleft \cdots \triangleleft H_{1}=$ $H^{m}$, where $G_{i+1}=\left[G_{i}, G_{i}\right]$ and $H_{i+1}=\left[H_{i}, H_{i}\right]$, for $1 \leqslant i \leqslant c$. By Lemma 3.2, there is an existential formula that defines $G^{m}$ in $G$ and $H^{m}$ in $H$. Also, for each integer $i>1$, there is an existential formula that defines $G_{i+1}$ in $G_{i}$ and $H_{i+1}$ in $H_{i}$. Using induction and Lemma 2.1-(iv), we see that, for each $i \in\{1, \ldots, c+1\}$, there is an existential formula $\phi^{i}$ such that $G_{i}=G_{\phi^{i}}$ and $H_{i}=H_{\phi^{i}}$.

By Lemma 3.3, we know that $G_{\phi^{i}} / G_{\phi^{i+1}} \simeq H_{\phi^{i}} / H_{\phi^{i+1}}$, for $1 \leqslant i \leqslant c$. The existence of these isomorphisms implies that $G_{\phi^{i}}=1$ if and only if $H_{\phi^{i}}=1$ and so, $H^{m}$ and $G^{m}$ are both soluble of class $c$.

CoRollary 3.5. Let $G$ and $H$ be polycyclic-by-finite groups such that $G \equiv_{1} H$. Then $G$ and $B$ have the same Hirsch number.

Proof: We follow the same notation of the preceding proposition. The groups $G / G^{m}$ and $H / H^{m}$ are finite and so $h\left(G / G^{m}\right)=h\left(H / H^{m}\right)=0$. Proposition 3.4 implies that

$$
h(G)=h\left(G^{m}\right)=\sum_{i=1}^{c} h\left(G_{\phi^{i}} / G_{\phi^{i+1}}\right)=\sum_{i=1}^{c} h\left(H_{\phi^{i}} / H_{\phi^{i+1}}\right)=h\left(H^{m}\right)=h(H) .
$$

Now, we can finally show that the hypothesis of Proposition 3.1 can be expressed by a formula.

Corollary 3.6. Let $G$ and $H$ be polycyclic-by-finite groups with $G \equiv_{1} H$ and let $r \geqslant 1$ be an integer. Suppose $\left\{g_{1}, \ldots, g_{k}\right\}$ is a set of generators of $G$. Then, there is an $\exists \forall$ formula $\gamma\left(X_{1}, \ldots, X_{k}\right)$ satisfying the following conditions:

(i) $\gamma\left(g_{1}, \ldots, g_{k}\right)$ is true in $G$;

(ii) if $B \vDash \gamma\left(h_{1}, \ldots, h_{k}\right)$ then $\left\langle h_{1}, \ldots, h_{k}\right\rangle$ is a subgroup of $B$ whose index is finite and prime to $r$.

Proof: By Proposition 3.4, there exist integers $m \geqslant 1, c \geqslant 1$ and existencial formulas $\phi^{1}, \ldots, \phi^{c+1}$, such that the derived series of $G^{m}$ and $H^{m}$ are

$$
1=G_{\phi^{c+1}} \triangleleft G_{\phi^{c}} \triangleleft \cdots \triangleleft G_{\phi^{1}}=G^{m} \quad \text { and } \quad 1=H_{\phi^{c+1}} \triangleleft H_{\phi^{c}} \triangleleft \cdots \triangleleft H_{\phi^{1}}=H^{m} \text {. }
$$

We write $\bar{X}=\left(X_{1}, \ldots, X_{k}\right)$. The group $G / G_{\phi^{1}}=G / G^{m}$ is finite and $G=$ $\left\langle g_{1}, \ldots, g_{k}\right\rangle G_{\phi^{1}}$. By Corollary 2.5, there exists an $\exists \forall$ formula, $\gamma_{0}(X)$, such that $G \vDash \gamma_{0}\left(g_{1}, \ldots, g_{k}\right)$ and, if $H \vDash \gamma_{0}\left(h_{1}, \ldots, h_{k}\right)$, then $H=\left\langle h_{1}, \ldots, h_{k}\right) H_{\phi^{2}}$.

By Lemma 2.1-(iv) and Lemma 3.2, for each $i \in\{1, \ldots, c\}$, there is an existential formula $\psi^{i}$ such that $\left(G_{\phi^{i}}\right)^{r}=G_{\psi^{i}}$ and $\left(H_{\phi^{i}}\right)^{r}=H_{\psi^{i}}$. The group $G_{\phi^{i}} / G_{\psi^{i}}$ is finite 
and $G_{\phi^{i}}$ is contained in $\left\langle g_{1}, \ldots, k\right\rangle G_{\psi^{i}}$. Therefore, Corollary 2.5 implies that there is an $\exists \forall$ formula $\gamma_{i}(X)$, such that: $G \vDash \gamma_{i}\left(g_{1}, \ldots, g_{k}\right)$ and, if $B \vDash \gamma_{i}\left(h_{1}, \ldots, h_{k}\right)$, then $H_{\phi^{i}} \subset\left\langle h_{1}, \ldots, h_{k}\right\rangle H_{\psi^{i}}$.

Denote by $\gamma(X)$ the $\exists \forall$ formula $\bigwedge_{i=0}^{c} \gamma_{i}(X)$. Clearly $G$ satisfies $\gamma\left(g_{1}, \ldots, g_{k}\right)$. Moreover, $H \vDash \gamma\left(h_{1}, \ldots, h_{k}\right)$ implies that $H=\left\langle h_{1}, \ldots, h_{k}\right\rangle H^{m}$ and that $H_{\phi^{i}}$ is contained in $\left\langle h_{1}, \ldots, h_{k}\right\rangle\left(H_{\phi^{i}}\right)^{r}$ for $1 \leqslant i \leqslant c$. So, (i) is proved and (ii) is a direct consequence of Proposition 3.1.

Following the notation of the Corollary above, we would like to have $H_{\boldsymbol{r}}=$ $\left\langle h_{1}, \ldots, h_{k}\right\rangle$ isomorphic to $G$. Using the above results, it is not dificult to obtain $H_{r}$ isomorphic to a quotient of $G$ by a finite normal subgroup (this is detailed in the theorem's demonstration). So, what we need is a formula that forces $H_{r}$ to be isomorphic to $G$, knowing that $H_{r} \simeq G / S$ with $|S|<\infty$. Finite subgroups can be described by formulas; consequently, we can oblige $H_{r}$ to contain a subgroup isomorphic to a certain finite subgroup of $G$. So, we are done if we have a finite subgroup $T_{G}$ of $G$ such that $T_{G} \hookrightarrow G / S$ with $S$ finite obliges $S=1$. Then, it is enough to take $T_{G}$ as the maximal finite normal subgroup of $G$.

DEFINITION: Given a polycyclic-by-finite group $G$, we shall note by $T_{G}$ the unique maximal finite normal subgroup of $G$. (The existence of such a group is guaranteed because $G$ satisfies the maximal condition on subgroups; uniqueness follows from the fact that the product of two finite normal subgroups is a finite normal subgroup).

\section{PROOF OF THE THEOREM}

Let $G$ and $H$ be polycyclic-by-finite groups and let $r \geqslant 1$ be an integer. As the hypothesis of the Theorem are symmetrical on $G$ and $H$, it is enough to show that there exists $H_{r}$ subgroup of $H$ such that $G \simeq H_{r}$ and $\left[H: H_{r}\right]$ is finite and prime to $r$. So, the Theorem is a consequence of the following two facts.

FACT 1. There is a subgroup $H_{T}$ of $H$ such that:

(i) $H_{r}$ is an homomorphic image of $G$;

(ii) $\left[H: H_{r}\right]$ is finite and prime to $r$;

(iii) there exists $T \triangleleft H$ such that $T \subset H_{r}$ and $T \simeq T_{G}$.

FACT2. If $B_{r}$ is a subgroup of finite index in $H$, satisfying conditions (i) and (iii) of Fact 1, then $\boldsymbol{H}_{\boldsymbol{r}} \simeq G$.

ProOF OF FACT 1: Consider a finite presentation of $G$ :

$$
G=\left\langle g_{1}, \ldots, g_{k}: R_{1}\left(g_{1}, \ldots, g_{k}\right)=\cdots=R_{l}\left(g_{1}, \ldots, g_{k}\right)=1\right\rangle
$$


From now on, we write $\bar{X}=\left(X_{1}, \ldots, X_{k}\right)$ and $\bar{g}=\left(g_{1}, \ldots, g_{k}\right)$. Let $\gamma(X)$ be the $\exists \forall$ formula given by Corollary 3.6 for the generators $g_{1}, \ldots, g_{k}$ of $G$ and for the integer $r$. We know that $G$ satisfies $\gamma(\bar{g})$. Suppose $\left\{x_{1}, \ldots, x_{n}\right\}=T_{G}$ and consider the universal formula $\rho_{T_{G}}\left(X_{1}, \ldots, X_{n}\right)$ given by Corollary 2.4. Let $u_{1}(\bar{X}), \ldots, u_{n}(\bar{X})$ be $n$ words such that $x_{i}=u_{i}(\bar{g})$, for $1 \leqslant i \leqslant n$. Then $G$ satisfies $\rho_{T_{G}}\left(\mu_{1}(\bar{g}), \ldots, \mu_{n}(\bar{g})\right)$.

Define the formula $\theta(\bar{X})$ as

$$
\left(\bigwedge_{i=1}^{l} R_{i}(\bar{X})=1\right) \wedge \gamma(\bar{X}) \wedge \rho_{T_{G}}\left(u_{1}(\bar{X}), \ldots, u_{n}(\bar{X})\right) .
$$

$G \vDash \theta(\bar{g})$ and $\theta(\bar{X})$ is an $\exists \forall$ formula. Since $G \equiv_{1} H$, the $\exists \forall$ sentence $(\exists \bar{X}) \theta(\bar{X})$ is satisfied in $H$. Let $h_{1}, \ldots, h_{k}$ be such that $H \vDash \theta\left(h_{1}, \ldots, h_{k}\right)$ and set $H_{r}=$ $\left\langle h_{1}, \ldots, h_{k}\right\rangle$. As $H \vDash\left(\bigwedge_{i=1}^{1} R_{i}\left(h_{1}, \ldots, h_{k}\right)=1\right)$, the map $g_{i} \rightarrow h_{i}$ induces a group homomorphism from $G$ to $H$. Since $H \vDash \gamma\left(h_{1}, \ldots, h_{k}\right)$, Corollary 3.6 implies that $\left[H: H_{r}\right]$ is finite and prime to $r$. As $B \vDash \rho_{T_{G}}\left(u_{1}\left(h_{1}, \ldots, h_{k}\right), \ldots, u_{n}\left(h_{1}, \ldots, h_{k}\right)\right)$, Corollary 2.4 implies that $T=\left\{u_{1}\left(h_{1}, \ldots, h_{k}\right), \ldots, u_{n}\left(h_{1}, \ldots, h_{k}\right)\right\}$ is a normal subgroup of $H$ isomorphic to $T_{G}$.

ProOF OF FACT 2: By (i), we can take $S \triangleleft G$ such that $G / S \simeq H_{r}$. By Corollary $3.5, G$ and $H$ have the same Hirsch number. Since $\left[H: H_{r}\right]$ is finite, we have $h(G)=$ $h\left(H_{r}\right)$ and so, $h(S)=0$, that is, $S$ is finite.

By (iii), there is $T \triangleleft H$ such that $T \simeq T_{G}$ and $T \subset H_{r}$. The isomorphism between $G / S$ and $H_{r}$, implies the existence of $T^{*} \triangleleft G$ such that $S \subset T^{*}$ and $T^{*} / S \simeq T_{G}$. Since $S$ and $T_{G}$ are finite, so is $T^{*}$; hence, $T^{*} \subset T_{G}$, by the maximality of $T_{G}$. Now we have $T_{G} \simeq T^{*} / S \subset T_{G} / S$ and so, $S=1$.

\section{Some equivalence Relations}

We begin by giving the definition of commensurability and giving a name for the equivalence relation that appears in the Theorem.

Definition: Two groups $G$ and $B$ are said to be commensurable if there exist $G_{*}$ and $H_{*}$, subgroups of $G$ and $H$ respectively, such that $G_{*} \simeq H_{*}$ and the indices $\left[G: G_{*}\right]$ and $\left[H: H_{*}\right]$ are finite. This notion has been introduced by Baumslag $[1$, p.9].

DEFINITION: Two groups $G$ and $H$ are said to be strongly commensurable if, for each $n>1$, there are $G_{n}$ and $H_{n}$, subgroups of $G$ and $H$ respectively, such that $G_{n} \simeq H, H_{n} \simeq G$ and the indices $\left[H: H_{n}\right]$ and $\left[G: G_{n}\right]$ are finite and prime to $n$.

Let $G$ and $H$ be polycyclic-by-finite groups and consider the following six equivalence relations:

(1) $G \times \mathbb{Z} \simeq H \times \mathbb{Z}$ 
(2) $G \equiv H$;

(3) $G \equiv_{1} B$;

(4) $G$ and $H$ strongly commensurable;

(5) $G$ and $H$ commensurable;

(6) $\mathcal{F}(G)=\mathcal{F}(H)$.

$(1) \Longrightarrow(2)$ is a result of Oger and holds in the class of groups (see [8]). An example showing that

(2) $\Longrightarrow(1)$ can be found in [9, Proposition].

(2) implies (3) is evident. To the best of the author's knowledge it is not known if (2) and (3) are equivalent.

$(3) \Longrightarrow(4)$ is the Theorem we have proved here and (4) $\Longrightarrow(3)$ is a consequence of the example at the end of this section.

$(4) \Longrightarrow(5)$ is clear from the definitions and $(4) \Longrightarrow(6)$ can be easily proved (a proof is given in next lemma).

Neither (5) implies (6) nor (6) implies (5) (see $[1$, p.9]). Consequently (4) is not implied by either (5) or (6).

Lemma 5.1. Suppose $G$ and $H$ are polycyclic-by-finite groups strongly commensurable. Then $\mathcal{F}(G)=\mathcal{F}(H)$.

Proof: It is enough to show that $\mathcal{F}(G) \subset \mathcal{F}(H)$. Moreover, we remark that $\mathcal{F}(G) \subset \mathcal{F}(H)$ if and only if, for each integer $m>1$, we have $G / G^{m} \in \mathcal{F}(H)$. The proof of this is straightforward once we use that $G / G^{m}$ and $H / H^{m}$ are finite.

For each integer $m>1$, there is $K<G$ such that $K \simeq H$ and $[G: K]$ is finite and prime to $m$. By the first paragraph, it is enough to show that $G / G^{m}$ is isomorphic to a quotient of $K$. If $p$ is a prime that divides $\left|G / G^{m}\right|$, there exists an element of order $p$ in $G / G^{m}$ and so, $p$ divides $m$. We conclude that $\left|G / G^{m}\right|$ is prime to $[G: K]$. As the index $\left[G: K G^{m}\right]$ divides $\left[G: G^{m}\right]$ and also divides $[G: K]$, we have $G$ equals to $K G^{m}$. Then $G / G^{m}$ is isomorphic to $K /\left(G^{m} \cap K\right)$.

EXAmple. Following [3], given a commutative ring $R$ with 1 and a faithfull $R$-module $M$, we define

$$
\Gamma(R, M)=\left\{\left(\begin{array}{ccc}
1 & r & n \\
0 & 1 & m \\
0 & 0 & 1
\end{array}\right) \quad / r \in R, m, n \in M\right\} .
$$

$\Gamma(R, M)$ is a group under the usual matrix product. We identify $\Gamma(R, M)$ to the set $R \times M \times M$ with multiplication defined by:

$$
(r, m, n)\left(r^{\prime}, m^{\prime}, n^{\prime}\right)=\left(r+r^{\prime}, m+m^{\prime}, r m^{\prime}+n+n^{\prime}\right) .
$$


We have $1_{\Gamma(R, M)}=(0,0,0)$ and $\left[(r, m, n),\left(r^{\prime}, m^{\prime}, n^{\prime}\right)\right]=\left(0,0, r m^{\prime}-r^{\prime} m\right)$. The centre $\zeta(\Gamma(R, M))$ and the derived subgroup $\Gamma(R, M)^{\prime}$ are both equal to $(0,0, M)$. So, $\Gamma(R, M)$ is nilpotent of class 2 .

Let $R$ be $\mathbb{Z}[\sqrt{5} i]$, the ring of algebraic integers in $\mathbb{Q}(\sqrt{5} i)$, and let $M$ be the $R$ ideal generated by 2 and $1+\sqrt{5} i$. Then $M$ is a non principal ideal of $R$. The group $\Gamma(R, R)$ is generated by $(1,0,0),(\sqrt{5} i, 0,0),(0,1,0)$ and $(0, \sqrt{5} i, 0)$. The group $\Gamma(R, M)$ is generated by $(1,0,0),(\sqrt{5} i, 0,0),(0,2,0)$ and $(0,1+\sqrt{5} i, 0)$. So, both groups are finitely generated and nilpotent, therefore polycyclic. Proposition $\mathrm{B}$ of [3] shows that $\Gamma(R, M)$ and $\Gamma(R, R)$ have the same finite images and are not isomorphic. Here we prove that $\Gamma(R, M)$ and $\Gamma(R, R)$ are strongly commensurable but they do not satisfy the same sentences with one alternation of quantifiers.

Lemma 5.2. Let $R$ be $\mathbb{Z}[\sqrt{5} i]$, the ring of algebraic integers of $\mathbb{Q}(\sqrt{5} i)$, and let $M$ be the ideal of $R$ generated by 2 and $1+\sqrt{5} i$. Then $\Gamma(R, M)$ and $\Gamma(R, R)$ do not satisfy the same sentences with one alternation of quantifiers.

Proof: Let $\sigma(X)$ be the universal formula $(\forall Y)([X, Y]=1)$. The formula $\sigma(x)$ is true in $\Gamma(R, R)$ (in $\Gamma(R, M)$ ) if and only if $x$ is in $\Gamma(R, R)^{\prime}$ (in $\Gamma(R, M)^{\prime}$ ). Now consider the $\forall \exists$ sentence $\left(\forall X_{1} \forall X_{2}\right)\left(\exists Y_{1} \ldots \exists Y_{4}\right) \phi\left(X_{1}, X_{2}, Y_{1}, \ldots, Y_{4}\right)$ where $\phi$ is given by:

$$
\begin{aligned}
& \bigwedge_{i=1,2}\left(\neg \sigma\left(Y_{i} Y_{i+2}\right) \wedge \neg \sigma\left(Y_{i} Y_{i+2}^{-1}\right)\right) \\
& \quad \wedge\left[Y_{1}, Y_{3}\right]=1 \wedge\left[Y_{2}, Y_{4}\right]=1 \wedge\left[Y_{1}, Y_{2}\right]=\left[X_{1}, X_{2}\right]\left[Y_{3}, Y_{4}\right]=\left[X_{1}, X_{2}\right]^{3}
\end{aligned}
$$

We shall show that this sentence is true in $\Gamma(R, M)$ but is not true in $\Gamma(R, R)$.

We first work in $\Gamma(R, M)$. Clearly $M$ is equal to $\{x+y \sqrt{5} i \in R \mid x+y$ is even $\}$. Moreover, for all $r \in R$, each of the complex numbers $r(1 \pm \sqrt{5} i) / 2$ belongs to $R$ if and only if $r$ belongs to $M$. We shall use these facts without stating. Given $x_{1}$ and $x_{2}$ in $\Gamma(R, M)$, the commutator $\left[x_{1}, x_{2}\right]$ is equal to $(0,0, a)$ with $a$ in $M$. So, $a(1 \pm \sqrt{5} i) / 2$ belongs to $R$. Consider the following elements of $\Gamma(R, M)$ :

$$
y_{1}=(1,0,0) y_{2}=(0, a, 0) \quad y_{3}=(a(1+\sqrt{5} i) / 2,0,0) \quad y_{4}=(0,1-\sqrt{5} i, 0) .
$$

We have $y_{3}^{-1}=(-a(1+\sqrt{5} i) / 2,0,0)$ and $y_{4}^{-1}=(0,-1+\sqrt{5} i, 0)$. Therefore, we know that

$$
\begin{array}{ll}
y_{1} y_{3}=(1+a(1+\sqrt{5} i) / 2,0,0) & y_{1} y_{3}^{-1}=(1-a(1+\sqrt{5} i) / 2,0,0) \\
y_{2} y_{4}=(0, a+1-\sqrt{5} i, 0) & y_{2} y_{4}^{-1}=(0, a-1+\sqrt{5} i, 0) .
\end{array}
$$


As $a$ belongs to $R$, either $1+a(1+\sqrt{5} i) / 2$ and $1-a(1+\sqrt{5} i) / 2$ are different from zero and so, $y_{1} y_{3} \notin \Gamma[R, M]^{\prime}$ and $y_{1} y_{3}^{-1} \notin \Gamma[R, M]^{\prime}$. Also if we have $a \neq-1+\sqrt{5} i$ and $a \neq 1-\sqrt{5} i$, we know that $y_{2} y_{4}$ and $y_{2} y_{4}^{-1}$ are not in $\Gamma[R, M]^{\prime}$. Direct computations show that

$$
\begin{aligned}
& {\left[y_{1}, y_{2}\right]=(0,0, a), \quad\left[y_{1}, y_{3}\right]=(0,0,0), \quad\left[y_{2}, y_{4}\right]=(0,0,0) \text { and }} \\
& {\left[y_{3}, y_{4}\right]=(0,0, a(1+\sqrt{5} i)(1-\sqrt{5} i) / 2)=(0,0,3 a)=(0,0, a)^{3}}
\end{aligned}
$$

So, we have proved that if $\left[x_{1}, x_{2}\right] \neq-1+\sqrt{5} i$ and $\left[x_{1}, x_{2}\right] \neq 1-\sqrt{5} i$, then $\phi\left(x_{1}, x_{2}, y_{1}, \ldots, y_{4}\right)$ is true in $\Gamma(R, M)$. In the case $\left[x_{1}, x_{2}\right]=-1+\sqrt{5} i$ or $\left[x_{1}, x_{2}\right]=$ $1-\sqrt{5} i$, we take

$$
z_{1}=(1,0,0) \quad z_{2}=(0, a, 0) \quad z_{3}=(a(1-\sqrt{5} i) / 2,0,0) \quad z_{4}=(0,1+\sqrt{5} i, 0),
$$

and we have that $\phi\left(x_{1}, x_{2}, z_{1}, \ldots, z_{4}\right)$ is true in $\Gamma(R, M)$. Consequently $\Gamma(R, M)$ satisfies the sentence $\left(\forall X_{1} \forall X_{2}\right)\left(\exists Y_{1} \ldots \exists Y_{4}\right) \phi\left(X_{1}, X_{2}, Y_{1}, \ldots, Y_{4}\right)$.

Now we work in $\Gamma(R, R)$. For $1 \leqslant i \leqslant 4$, take $w_{i}=\left(w_{i 1}, w_{i 2}, w_{i 3}\right)$ in $\Gamma(R, R)$ and call $\overline{w_{i}}$ the element $\left(w_{i 1}, w_{i 2}\right)$ in $R \times R$. Then $\left[w_{i}, w_{j}\right]$ is equal to $\left(0,0, \operatorname{det}\left(\overline{w_{i}}, \overline{w_{j}}\right)\right)$. Suppose that $\left[w_{1}, w_{2}\right]=(0,0,1),\left[w_{3}, w_{4}\right]=(0,0,3)$ and $\left[w_{1}, w_{3}\right]=\left[w_{2}, w_{4}\right]=(0,0,0)$. We have $\operatorname{det}\left(\overline{w_{1}}, \overline{w_{3}}\right)=\operatorname{det}\left(\overline{w_{2}}, \overline{w_{4}}\right)=0$ and so, there are $\alpha_{13}$ and $\alpha_{24}$ in $\mathbb{Q}(\sqrt{5} i)$ such that $\overline{w_{3}}=\alpha_{13} \overline{w_{1}}$ and $\overline{w_{4}}=\alpha_{24} \overline{w_{2}}$. As $\left[w_{3}, w_{2}\right]$ is in $(0,0, R)$, we know that $\operatorname{det}\left(\overline{w_{3}}, \overline{w_{2}}\right)$ is in $R$; moreover, $\operatorname{det}\left(\overline{w_{1}}, \overline{w_{2}}\right)=1$ implies $\alpha_{13}=\operatorname{det}\left(\overline{w_{3}}, \overline{w_{2}}\right)$ and so, $\alpha_{13}$ is in $R$. The same argument shows that $\alpha_{24}$ is in $R$. Since $\left[w_{3}, w_{4}\right]=(0,0,3)$, we have:

$$
3=\operatorname{det}\left(\overline{w_{3}}, \overline{w_{4}}\right)=\alpha_{13} \alpha_{24} \operatorname{det}\left(\overline{w_{1}}, \overline{w_{2}}\right)=\alpha_{13} \alpha_{24} .
$$

As $\alpha_{13}$ and $\alpha_{24}$ are in $R$ and $\alpha_{13} \alpha_{24}=3$, we conclude that either $\alpha_{13}= \pm 1$ or $\alpha_{24}= \pm 1$. So, we have either $\overline{w_{3}}= \pm \overline{w_{1}}$ or $\overline{w_{4}}= \pm \overline{w_{2}}$. Since $\left(u_{1}, u_{2}, u_{3}\right)^{-1}=$ $\left(-u_{1},-u_{2}, u_{1} u_{2}-u_{3}\right)$ for every $\left(u_{1}, u_{2}, u_{3}\right)$ in $\Gamma(R, R)$, we conclude that one of the four elements $w_{1} w_{3}, w_{1} w_{3}^{-1}, w_{2} w_{4}$ or $w_{2} w_{4}^{-1}$ must be in $(0,0, R)=\Gamma(R, R)^{\prime}$. What we have proved above is that if $w_{1}, w_{2}, w_{3}$ and $w_{4}$ are elements of $\Gamma(R, R)$ such that $\left[w_{1}, w_{2}\right]=(0,0,1),\left[w_{3}, w_{4}\right]=(0,0,3)$ and $\left[w_{1}, w_{3}\right]=\left[w_{2}, w_{4}\right]=(0,0,0)$, then one of the four elements $w_{1} w_{3}, w_{1} w_{3}^{-1}, w_{2} w_{4}$ or $w_{2} w_{4}^{-1}$ must be in $\Gamma(R, R)^{\prime}$. This shows that if we put $x_{1}=(1,0,0)$ and $x_{2}=(0,1,0)$ then $\left[x_{1}, x_{2}\right]=(0,0,1)$ and, for any $y_{1}, \ldots, y_{4}$ in $\Gamma(R, R)$, the formula $\phi\left(x_{1}, x_{2}, y_{1}, \ldots, y_{4}\right)$ is not true in $\Gamma(R, R)$.

LEммA 5.3. Let $R$ be the ring of algebraic integers of a finite Galois-extention of $\mathbb{Q}$. Let $M$ and $N$ be non zero ideals of $R$. Then $\Gamma(R, M)$ and $\Gamma(R, N)$ are strongly commensurable. 
Proof: By the hypothesis of simmetry, it is enough to show that for each integer $m>1$, there exists a subgroup $H$ of $\Gamma(R, N)$ such that $H \simeq \Gamma(R, M)$ and the index $[\Gamma(R, N): H]$ is finite and prime to $m$. We first prove the following fact:

FACT (A). For each integer $m>1$, there is an integer $k$ which is prime to $m$ and there is an ideal $M^{\prime}$ of $R$ such that $M^{\prime} \simeq M$ and $k N \subset M^{\prime} \subset N$.

Consider the ring $R_{m}=\{r / n: r \in R$ and $n \in \mathbb{Z}$ is prime to $\mathrm{m}\}$. Since $R$ is a Dedekind domain, so is $R_{m}$. Moreover, $R_{m}$ is a principal domain because it has a finite number of prime ideals ( see [4, Corollary on page 13]). Consequently, there exists a $R_{m}$ isomorphism $f: R_{m} M \longrightarrow R_{m} N$. As $M$ and $N$ are finitely generated $R$-ideals, there exists an integer $t \geqslant 1$ which is prime to $m$ and such that $t f(M) \subset N$ and $t f^{-1}(N) \subset M$. Therefore, $f^{-1}\left(t^{2} N\right) \subset t M$ and this implies $t^{2} N \subset f(t M)=t f(M) \subset N$. Setting $k=t^{2}$ and $M^{\prime}=t f(M)$, we obtain (A).

Now, put $H=\Gamma\left(R, M^{\prime}\right)$. It remains to prove that the index $[\Gamma(R, N): H]$ is prime to $m$. Direct computations show that if $L$ is an $R$-submodule of $N$ then the index $[\Gamma(R, N): \Gamma(R, L)]$ is equal to $[N: L]^{2}$. Hence, it is enough to show that $\left[N: M^{\prime}\right]$ is prime to $m$. By (A), we know that $\left[N: M^{\prime}\right]$ divides $[N: k N]$; moreover, the index [N:kN] is prime to $m$ because it has the same prime divisors as $k$. So, $\left[N: M^{\prime}\right]$ is prime to $m$.

\section{REFERENCES}

[1] G. Baumslag, Lecture notes on nilpotent groups, Regional Conference Series in Math., 2 (American Mathematical Society, Providence, RI, 1971).

[2] C.C. Chang and H.J. Keisler, Model theory (North-Holland, Amsterdam, New York, 1973).

[3] F.J. Grunewald and R. Scharlau, 'A note on finitely generated torsion-free nilpotent groups of class 2', J. Algebra 58 (1979), 162-175.

[4] S. Lang, Algebraic number theory, Graduate Texts in Math 110 (Springer Verlag, Berlin, Heidelberg, New York, 1986).

[5] F. Oger, 'Elementary equivalence and genus of finitely generated nilpotent groups', Bull. Austral. Math. Soc. 37 (1988), 61-68.

[6] F. Oger, 'Équivalence élémentaire entre groupes finis-par-abéliens de type fini', Comment. Math. Helv. 57 (1982), 469-480.

[7] F. Oger, 'Cancellation and elementary equivalence of finitely generated finite-by-nilpotent groups', J. London Math. Soc. 44 (1990), 173-183.

[8] F. Oger, 'Cancellation and elementary equivalence of groups', J. Pure Appl. Algebra 30 (1983), 293-299.

[9] F. Oger, 'Elementary equivalence and profinite completions: a characterization of finitely generated abelian-by-finite groups', Proc. Amer. Math. Soc. 103 (1988), 1041-1048.

[10] A.H. Rhemtulla, 'Commutators of certain finitely generated soluble groups', Canad. J. Math. 21 (1969), 1160-1164. 
[11] V.A. Romankov, 'Width of verbal subgroups in solvable groups', Algebra and Logic 21 (1982), 41-49.

[12] D. Segal, Polycyclic groups (Cambridge University Press, Cambridge, 1983).

Universidade de São Paulo

Instituto de Matemática e Estatística

Caixa Postal 66281 - CEP 05389-970

São Paulo - Brasil 